\title{
IMPLANTAÇÃO DE AÇÕES PARA ENFRENTAMENTO DO CONSUMO DE DROGAS NA ATENÇÃO PRIMARIA A SAÚDE*
}

\author{
Jessica Adrielle Teixeira Santos ${ }^{1}$, Magda Lúcia Félix de Oliveira ${ }^{2}$
}

\begin{abstract}
RESUMO: O estudo avaliou a implantação de diretrizes para atenção integral a usuários de álcool e outras drogas na Atenção Primária à Saúde, por meio de pesquisa avaliativa de estudo de caso, utilizando-se a diretriz do Ministério da Saúde para esta política pública. Participaram gestores municipais e profissionais de saúde de uma Unidade Básica de Saúde, sendo a maioria mulheres com idade entre 30 e 49 anos e tempo no cargo/função inferior a cinco anos. Como julgamento preconizou-se que a proporção de respostas concordantes com a diretriz corresponderia ao grau de implantação: implantada $(>80 \%)$, insatisfatória $(40 \%$ a $79,9 \%)$ e crítica $(<39,9 \%)$. O grau de implantação global das diretrizes foi insatisfatório $(66,9 \%)$, no entanto, para os gestores $(91,6 \%)$ e alguns profissionais $(80,5 \%)$ este foi considerado aceitável. Os resultados apontam a necessidade de analisar o papel de diferentes equipamentos que compõem a rede de atenção integral.

DESCRITORES: Avaliação; Políticas públicas de saúde; Usuários de drogas; Atenção primária a saúde.

\section{IMPLANTATION OF ACTIONS FOR CONFRONTING DRUG CONSUMPTION IN PRIMARY HEALTH CARE}

\begin{abstract}
The study assessed the implantation of directives for integral care of users of alcohol and other drugs in Primary Health Care, through evaluative research case studies, using the Ministry of Health directives for this public policy. City managers and health professionals from a Primary Care center participated, the majority of whom were women aged between 30 and 49 years, who had spent less than five years in their current positions/ roles. As a parameter, it was recommended that the proportion of answers consistent with the directive would correspond to the extent of implantation: implanted (>80\%), dissatisfactory $(40 \%$ to $79.9 \%)$ and critical $(<39.9 \%)$. The global extent of implantation of the directives was dissatisfactory $(66.9 \%)$, however, for the managers $(91.6 \%)$ and some health professionals $(80.5 \%)$ this was considered acceptable. The results indicate the need to analyze the role of different equipment which are part of the integral care network.
\end{abstract}

DESCRIPTORS: Assessment; Public health policies; Drug users; Primary health care.

\section{IMPLANTAÇÃO DE AÇÕES PARA ENFRENTAMENTO DO CONSUMO DE DROGAS NA ATENCIÓN PRIMARIA A SALUD}

RESUMEN: Este estudio evaluó la implantación de directrices para atención integral a usuarios de álcohol y otras drogas en la Atención Primaria a la Salud, por medio de investigación evaluativa de estudio de caso, utilizándose la directriz del Ministerio de la Salud para esta política pública. Participaron gestores municipales y profesionales de salud de una Unidad Básica de Salud, siendo la mayoría mujeres con edad entre 30 y 49 años y tiempo en el cargo/función inferior a cinco años. Como juzgamiento se preconizó que la proporción de respuestas que concuerdan con la directriz correspondería al grado de implantación: implantada $(>80 \%)$, insatisfactoria $(40 \%$ a $79,9 \%$ ) y crítica $(<39,9 \%)$. El grado de implantación global de las directrices fue insatisfactorio $(66,9 \%)$, sin embargo, para los gestores $(91,6 \%)$ y algunos profesionales $(80,5 \%)$ este fue considerado aceptable. Los resultados apuntan la necesidad de analizar el papel de diferentes equipos que componen la red de atención integral.

DESCRIPTORES: Evaluación; Políticas públicas de salud; Usuarios de drogas; Atención primaria a la salud.

*Estraído da dissertação apresentada à Universidade Estadual de Maringá-Paraná em 2011, subsidiada pela Fundação de Apoio ao Desenvolvimento Científico e Tecnológico do Paraná - Fundação Araucária.

${ }^{1}$ Enfermeira. Doutoranda em Enfermagem Psiquiátrica pela Escola de Enfermagem de Ribeirão Preto - Universidade de São Paulo. ${ }^{2}$ Enfermeira. Doutora em Saúde Coletiva. Professora do Departamento de Enfermagem da Universidade Estadual de Maringá.

Autor correspondente:

Jessica Adrielle Teixeira Santos

Universidade de São Paulo

R. Monte Alverne, 884 - 14050-120 - Ribeirão Preto-SP-Brasil

E-mail: jessica_drielle@yahoo.com.br
Recebido: 17/09/2012 Aprovado: 11/11/2012 


\section{INTRODUÇÃO}

O uso de drogas de abuso configura-se atualmente como um problema de saúde publica global, e de grande preocupação social. Resultados de diferentes pesquisas identificam o aumento do consumo de drogas de abuso para fins recreativos, a redução da idade de iniciação e a facilidade de sua obtenção, devido a diferentes formas de produção e preços mais acessíveis ${ }^{(1-2)}$. Cerca de 200 milhões de pessoas - quase $5 \%$ da população mundial entre 15 e 64 anos - usam drogas ilícitas pelo menos uma vez por ano $^{(3)}$. De acordo com o II Levantamento Domiciliar sobre o uso de drogas psicotrópicas em indivíduos de 12 a 65 anos nas 108 maiores cidades brasileiras, $22,8 \%$ dos entrevistados declararam ter usado algum tipo de droga no ano ${ }^{(4)}$.

Diante do padrão contemporâneo de consumo de drogas de abuso no Brasil a intervenção do Ministério da Saúde se concretizou mediante a formulação e implementação de portarias e resoluções normativas, incluindo o documento 'A Política do Ministério da Saúde para Atenção Integral a Usuários de Álcool e Outras Drogas', diretriz principal da Saúde Pública, publicada em $2004^{(5)}$. Essa política é convergente com os princípios e orientações do Sistema Único de Saúde - SUS, buscando a universalidade do acesso, integralidade das ações, equidade e direito à assistência a saúde aos usuários. Para a consecução de tais objetivos, a política está organizada a partir do estabelecimento de uma rede de atenção aos usuários de álcool e outras drogas, de forma integral e intersetorial ${ }^{(5)}$.

Apesar da existência de ampla rede de atenção, documentos oficiais da última década ratificam a ênfase do provimento do cuidado e atenção à saúde de usuários de álcool e outras drogas de abuso na atenção primária, por ser este um passo fundamental para o acesso e acolhimento de pacientes e suas famílias aos serviços de saúde e por considerar a resolutividade nesse nível de atenção ${ }^{(5-8)}$.

Nesta perspectiva, a Atenção Primária à Saúde (APS), atualmente desenvolvida por meio da Estratégia Saúde da Família (ESF) e considerada a 'porta de entrada' do SUS, é o local que deveria assumir, gradativamente, a posição de coordenador da assistência aos usuários de álcool e outras drogas ${ }^{(9)}$, graças às suas características básicas ${ }^{(10)}$.

Apesar de seu instrumental aparentemente simples, as ações desenvolvidas na APS são complexas ao nível das 'tecnologias humanas', pois envolvem sujeitos e objetos de natureza diferentes, situando a avaliação como necessidade operacional para a manutenção da qualidade e, também, como um desafio, devido à complexidade de seus processos ${ }^{(11-12)}$.

Assim, o presente estudo objetivou avaliar a implantação das diretrizes do documento 'A Política do Ministério da Saúde para Atenção Integral a Usuários de Álcool e Outras Drogas' na Atenção Primária à Saúde.

\section{MÉTODO}

Trata-se de uma pesquisa avaliativa, desenvolvida por meio de um estudo de caso único, em um município da Região Noroeste do Paraná - Brasil, cuja abordagem metodológica incorpora a avaliação da implantação de política pública voltada à atenção integral a usuários de álcool e outras drogas na atenção primária à saúde ${ }^{(13-14)}$.

Como padrão para orientar o processo avaliativo elegeu-se as diretrizes do documento A Política do Ministério da Saúde para a Atenção Integral à Usuários de Álcool e Outras Drogas - Intersetorialidade e Atenção Integral - Promoção e Proteção à saúde e de Prevenção de Agravos $^{(5)}$.

A partir do padrão estabelecido elaborou-se graficamente o modelo lógico de avaliação ${ }^{(14)}$, pautado na construção de 'eventos teoricamente previstos', que serviram como critérios para comparação dos eventos realizados e baseou-se na apresentação dos critérios avaliativos de forma sistematizada, os quais estão apresentados na tabela 1.

Escolheu-se, intencionalmente, uma UBS da região norte do município estudado, que possui em sua área de abrangência os maiores indicadores municipais de internação e violência relacionados à drogas de abuso, além de ser considerada de baixo desenvolvimento social ${ }^{(15-16)}$.

Para a realização do estudo elegeu-se, como usuários potenciais das diretrizes avaliadas, profissionais atuantes no sistema municipal e na unidade em 2011, abaixo discriminados:

- Gestores: gerente da Diretoria de Assistência e Promoção a Saúde, coordenador da Atenção Básica e diretor da Diretoria de Programas sobre Drogas $(\mathrm{n}=3)$.

- Profissionais das equipes de Saúde da Família da UBS: três médicos, três enfermeiros, três auxiliares de enfermagem, e 15 agentes comunitários de saúde - ACS $(\mathrm{n}=24)$.

- Profissionais de saúde do Núcleo de Apoio à Saúde da Família - NASF: um assistente social, um nutri- 
Tabela 1- Modelo lógico da avaliação das ações na Atenção Primária à Saúde*. Maringá, 2011

\begin{tabular}{|c|c|}
\hline \multicolumn{2}{|r|}{ Diretrizes e critérios avaliativos das ações na atenção primária à saúde } \\
\hline Intersetorialidade & $\begin{array}{l}\text { 1. Tema transversal que requer intensa capilaridade com outras áreas de conhecimento. } \\
\text { 2. Articular ações com a sociedade civil, movimentos sindicais, associações e orga- } \\
\text { nizações comunitárias. } \\
\text { 3. Requer investimentos contínuos, formulação de alternativas de sustentabilidade e } \\
\text { de financiamento das ações. } \\
\text { 4. Geração de conhecimento, informação e inovações científicas em parceria com } \\
\text { universidades. }\end{array}$ \\
\hline $\begin{array}{l}\text { Atenção Integral: } \\
\text { Prevenção de Agravos }\end{array}$ & $\begin{array}{l}\text { 1. A redução de danos deve ser considerada como estratégica ao planejamento de } \\
\text { propostas e ações preventivas. } \\
\text { 2. Capacitação e formação dos profissionais de saúde em uma perspectiva multiprofissional. } \\
\text { 3. Redução dos fatores de vulnerabilidade e risco específicos, e fortalecimento dos } \\
\text { fatores de proteção. } \\
\text { 4. Práticas preventivas devem ser planejadas levando em conta a especificidade ine- } \\
\text { rente a cada parcela populacional. }\end{array}$ \\
\hline $\begin{array}{l}\text { Atenção Integral: } \\
\text { Promoção e Proteção } \\
\text { à Saúde }\end{array}$ & $\begin{array}{l}\text { 1. Promover assistência em níveis de cuidados primários. } \\
\text { 2. Estruturação das redes assistenciais em saúde. } \\
\text { 3. Melhorar o acesso a dispositivos de cuidados e à rede de suporte social existente } \\
\text { em seu território. } \\
\text { 4. Potencializar o envolvimento familiar para o enfrentamento da vulnerabilidade } \\
\text { das pessoas. }\end{array}$ \\
\hline
\end{tabular}

*Adaptado do documento a Política do Ministério da Saúde para a Atenção Integral à Usuários de Álcool e Outras Drogas

cionista, um educador físico, um farmacêutico, e um psicólogo $(\mathrm{n}=5)$.

Após exclusão de 12 profissionais, quatro por estarem afastados das atividades e 9 recusas, restaram 19 participantes (61\%), que responderam questionário autoaplicável.

Para coleta de dados foram utilizados dois modelos de questionário autoaplicável, com questões descritivas, obedecendo às diretrizes e os critérios avaliativos do modelo lógico de avaliação, um direcionado aos gestores e outro direcionado aos profissionais de saúde da ESF.

Como critério de julgamento preconizou-se que a proporção de respostas que apresentassem consistência entre as propostas do documento da Política, corresponderia ao grau de implantação ${ }^{(17-18)}$, não existindo critérios de peso entre as questões (Quadro 1).

Para o tratamento dos dados, foi construída uma matriz de análise no programa Microsoft Excel. O grau de implantação global das diretrizes e o grau de implantação de cada diretriz foram obtidos após a soma dos percentuais de frequência, seguida do calculo da média aritmética, e apresentados sob a forma de tabelas com distribuição simples de frequência.
O projeto de pesquisa foi aprovado pelo Comitê de Ética e Pesquisa com Seres Humanos da Universidade Estadual de Maringá (Parecer n. 102/2011).

Quadro 1- Parâmetros para a classificação do grau de implantação da Política do Ministério da Saúde para a Atenção Integral à Usuários de Álcool e Outras Drogas. Maringá, 2011

\begin{tabular}{|l|l|}
\hline Grau de Implantação & $\begin{array}{l}\text { Percentual de } \\
\text { Concordância }\end{array}$ \\
\hline Implantação aceitável & Maior ou igual a 80\% \\
\hline Implantação insatisfatória & De 40 a 79,9\% \\
\hline Implantação crítica & Menor ou igual a 39,9\% \\
\hline
\end{tabular}

\section{RESULTADOS}

Entre os participantes, a maioria era do sexo feminino, com idade entre 30 a 49 anos (73,6\%), sendo que a equipe do NASF era composta apenas por mulheres, e a maioria tinha idade inferior a 29 anos. Quanto ao tempo no cargo/função, o período de um a cinco anos foi apontado por 9 participantes $(47,3 \%)$ e o período inferior a um ano, por com cinco $(26,3 \%)$, ou seja, a maioria atuava a período inferior a cinco anos no cargo/função. 
Conhecimento do documento A Política do Ministério da Saúde para Atenção Integral a Usuário de Álcool e Outras Drogas

Entre os entrevistados, dois referiram conhecer o documento, sendo eles, o diretor da Diretoria de Programas Sobre Drogas e a coordenadora da Atenção Básica. Cinco participantes referiram ter recebido informações/orientações genéricas sobre o mesmo, e manifestaram opiniões sobre o documento e apresentaram como ponto de convergência a falta de orientações para a prática.

Para os gestores, a política é convergente com os princípios e orientações do Sistema Único de Saúde e, apesar de algumas limitações, tem orientado a organização da rede de atenção à usuários de álcool e outras drogas.

Grau de implantação global e grau da implantação segundo as diretrizes e critérios avaliativos

O grau de implantação das diretrizes do documento do Ministério da Saúde foi verificado em um primeiro momento da forma global. Em um segundo momento, de acordo com as diretrizes e critérios avaliativos (Tabela 2), estabelecendo em paralelo a exemplificação das respostas dos participantes do estudo, para cotejar e dar sustentação à discussão dos resultados.

Tabela 2- Distribuição da frequência dos percentuais de concordância e dos critérios avaliativos. Maringá, 2011

\begin{tabular}{|c|c|c|c|c|c|c|c|c|}
\hline \multirow{2}{*}{ Diretrizes/Critérios } & \multicolumn{2}{|c|}{$\begin{array}{l}\text { GESTOR } \\
(\mathbf{n}=\mathbf{3})\end{array}$} & \multicolumn{2}{|c|}{$\begin{array}{c}\text { ESF* }^{*} \\
(n=13)\end{array}$} & \multicolumn{2}{|c|}{$\begin{array}{c}\text { NASF** }^{* *} \\
(\mathrm{n}=3)\end{array}$} & \multicolumn{2}{|c|}{$\begin{array}{l}\text { MUNICÍPIO } \\
\quad(n=19)\end{array}$} \\
\hline & n & $\%$ & n & $\%$ & $\mathbf{n}$ & $\%$ & n & $\%$ \\
\hline \multicolumn{9}{|l|}{ INTERSETORIALIDADE } \\
\hline $\begin{array}{l}\text { Capilaridade com Justiça, Educação, } \\
\text { Assistência Social e Desenvolvimento }\end{array}$ & 3 & 100,0 & 6 & 46,1 & 2 & 66,6 & 11 & 57,8 \\
\hline Articulação com sociedade civil & 3 & 100,0 & 7 & 53,8 & 2 & 66,6 & 12 & 63,1 \\
\hline Investimentos contínuos & 3 & 100,0 & 9 & 69,2 & 3 & 100,0 & 15 & 78,9 \\
\hline $\begin{array}{l}\text { Geração de conhecimento, informação e } \\
\text { inovação em parceria com Universidade }\end{array}$ & 3 & 100,0 & 8 & 61,5 & 2 & 66,6 & 13 & 68,4 \\
\hline GRAU DE IMPLANTAÇÃO & - & 100,0 & - & 57,6 & - & 74,9 & - & 67,0 \\
\hline \multicolumn{9}{|c|}{ ATENÇÃO INTEGRAL: PREVENÇÃO DE AGRAVOS } \\
\hline Estratégia de redução de danos & 3 & 100,0 & 3 & 23 & - & - & 6 & 31,5 \\
\hline $\begin{array}{l}\text { Capacitação e formação dos } \\
\text { profissionais }\end{array}$ & 3 & 100,0 & 7 & 53,8 & 3 & 100,0 & 13 & 68,4 \\
\hline $\begin{array}{l}\text { Redução dos fatores de vulnerabili- } \\
\text { dade e risco }\end{array}$ & 3 & 100,0 & 9 & 69,2 & 3 & 100,0 & 15 & 78,4 \\
\hline $\begin{array}{l}\text { Praticas preventivas com especificidade } \\
\text { à parcela populacional }\end{array}$ & 3 & 100,0 & 10 & 79,9 & 3 & 100,0 & 16 & 84,2 \\
\hline GRAU DE IMPLANTAÇÃO & - & 100,0 & $\sim$ & 56,4 & - & 75,0 & - & 65,6 \\
\hline \multicolumn{9}{|c|}{ ATENÇÃO INTEGRAL: PROMOÇÃO E PROTEÇÃO A SAÚDE } \\
\hline $\begin{array}{l}\text { Promoção de assistência em nível de } \\
\text { cuidados primários }\end{array}$ & 3 & 100,0 & 9 & 69,2 & 2 & 66,6 & 14 & 73,6 \\
\hline $\begin{array}{l}\text { Estruturação da rede assistencial em } \\
\text { saúde }\end{array}$ & 3 & 100,0 & 7 & 76,9 & 3 & 100,0 & 13 & 68,4 \\
\hline $\begin{array}{l}\text { Acessibilidade a rede de saúde e } \\
\text { suporte social }\end{array}$ & - & - & 8 & 61,5 & 3 & 100,0 & 11 & 57,8 \\
\hline Potencializar o envolvimento familiar & 3 & 100,0 & 8 & 61,5 & 3 & 100,0 & 14 & 73,6 \\
\hline GRAU DE IMPLANTAÇÃO & - & 75,0 & - & 67,2 & - & 91,6 & - & 68,3 \\
\hline $\begin{array}{l}\text { GRAU DE IMPLANTAÇÃO } \\
\text { GLOBAL }\end{array}$ & - & 91,6 & - & 60,4 & - & 80,5 & - & 66,9 \\
\hline
\end{tabular}

*ESF: Equipe Saúde da Família; **NASF: Núcleo de Apoio à Saúde da Família. 
Os resultados da avaliação apontam que o grau de implantação das diretrizes para enfrentamento do consumo de álcool e outras drogas de abuso na APS do município em estudo foi insatisfatório (percentual de concordância $=66,9 \%$ ), com variação de $65,6 \%$ para a diretriz Prevenção de Agravos e de 68,3\% para a diretriz Promoção e Proteção à Saúde. Para os gestores municipais e os profissionais de saúde do NASF, o grau de implantação foi considerado aceitável, com percentuais de concordância de $91,6 \%$ e 80,5\%, respectivamente. Entretanto, para os profissionais de saúde das equipes de ESF, executores diretos do cuidado à saúde nos aspectos de promoção e proteção à saúde e prevenção de agravos, o percentual de concordância foi de $60,4 \%$, correspondendo a um grau de implantação insatisfatório.

A análise do grau de Implantação das Diretrizes segundo os gestores e executores do cuidado apontou pior percentual de concordância para a diretriz Promoção e Proteção à Saúde. Para os gestores (75\%), a diretriz Intersetorialidade, para os profissionais do NASF (74, 9\%), e a diretriz Prevenção de Agravos para os profissionais das equipes de ESF. O reconhecimento da Intersetorialidade como uma diretriz para atenção integral a usuários de álcool e outras drogas obteve grau de implantação insatisfatório, com percentual de concordância de $67 \%$ das respostas; somente para os gestores o grau de implantação foi considerado aceitável $(100 \%)$.

A maioria dos profissionais da APS $(n=9)$ apontou que essas ações, quando presentes, materializam-se por meio de parcerias entre a unidade de saúde, as escolas e o Centro de Referência Assistência Social (CRAS) local, por meio da realização de atividades de prevenção e promoção da saúde, como palestras, orientações e atividades recreativas.

Com relação aos Investimentos Contínuos em Ações de Enfrentamento do uso de álcool e outras drogas, os profissionais de saúde referiram perceber o apoio por meio da distribuição de materiais educativos e informativos, capacitação e incentivos para realização de campanhas. Porém, quando se trata de recursos municipais, os gestores concordam com a escassez de recursos financeiros.

A diretriz Prevenção de Agravos atingiu concordância de 65,6\%, sendo avaliada com grau de implantação geral insatisfatório, exceto pelos gestores, cujas respostas, novamente, apresentaram percentual de concordância de $100 \%$. Os critérios avaliativos da diretriz Prevenção de Agravos também apresentaram padrões diferenciados de concordância: para os gestores todos os critérios tiveram concordância $100 \%$; para os profissionais das equipes de ESF nenhum critério foi considerado satisfatório; entre os profissionais do NASF três critérios apresentaram $100 \%$ de concordância, apenas o critério Estratégias de Redução de Danos não foi respondido por nenhum deles.

O grau de implantação global atingido pela diretriz Promoção e Proteção da Saúde foi insatisfatória, com percentual de concordância de $66,9 \%$, sendo avaliada como aceitável somente para os profissionais da equipe do NASF (grau de concordância de 91,6\%). Para os gestores e profissionais de saúde das equipes da ESF a diretriz foi considerada com implantação insatisfatória, com concordância de $75 \%$ e $67,2 \%$, respectivamente.

Apesar da Promoção de Assistência à Saúde em Nível de Cuidados Primários, ser considerada insatisfatória, profissionais da equipe do NASF $(n=2)$ e profissionais das equipes de $\operatorname{ESF}(n=7)$ relataram procurar envolver o indivíduo em uso/abuso de drogas com os cuidados oferecidos na Unidade.

Os profissionais que negaram Promover a Assistência aos Usuários de Álcool e Outras Drogas na Unidade ( $\mathrm{n}=8)$, justificaram elencando uma série de limitantes, como, falta de tempo para planejamento das ações, estrutura física da unidade, recusa do usuário de álcool e outras drogas, demanda da unidade sobrecarregada, e não aceitação da dependência pelo usuário, principalmente em uso/abuso de álcool. A falta de comunicação com o CAPSad de referência foi um problema citado por dois ACS. Com relação à Acessibilidade à Rede de Saúde e Suporte Social Local, todos os gestores concordam com as limitações do município para continuidade do tratamento.

No Contexto Organizacional, todos os profissionais de saúde do NASF $(n=3)$ e 8 profissionais das equipes de ESF afirmaram buscar facilitar o acesso ao serviço de saúde e à rede de suporte social existente no município, e em sua área de abrangência para indivíduos em uso/abuso de drogas. Todos os profissionais do NASF $(\mathrm{n}=3)$ e oito profissional de saúde da equipe de ESF procuram o Envolvimento Familiar como um todo nas ações de enfrentamento ao consumo de drogas de abuso desenvolvidas na Unidade.

\section{DISCUSSÃO}

Os participantes deste estudo refletem os recursos humanos do sistema de saúde brasileiro, cujos profissionais de saúde em sua maioria são mulheres, na faixa etária de 31 a 45 anos, e com tempo no cargo/função inferior ou igual a cinco anos ${ }^{(19)}$. 
Em 2004, o Ministério da Saúde divulgou o documento A Política do Ministério da Saúde para a Atenção Integral a Usuários de Álcool e Outras Drogas $^{(5)}$, distribuiu exemplares e orientou a divulgação das suas diretrizes, com incentivo à capacitação dos profissionais de saúde e da assistência social para o cumprimento dos objetivos. Entretanto, a realidade analisada evidencia a fragilidade dessa ação.

Ademais, é preciso pontuar que conhecimento e informação são termos comumente utilizados como sinônimos, no entanto, não têm o mesmo significado. $\mathrm{O}$ conhecimento é definido como o ato ou efeito de apreender intelectualmente determinado assunto, com ênfase do desenvolvimento teórico e prático, e, a informação é definida como um conhecimento obtido por meio de instrução, esclarecimento, indicação, comunicação ${ }^{(20)}$.

Percebe-se que o domínio teórico deste Documento fica mais concentrado nos núcleos gerenciais, com pouca disseminação para o campo da prática, onde se encontram os executores do cuidado, que poderiam, também, contribuir para o planejamento das ações, avaliação de intervenções ${ }^{(5)}$. Quando presentes correspondem à informações/orientações de caráter generalista, sem definição clara sobre suas fontes e/ou orientações práticas.

Considerando a incipiência das políticas públicas direcionadas ao enfrentamento do consumo de drogas de abuso, pressupõe-se que a não operância de algumas destas diretrizes não significa inviabilidade política, mas um período de 'janela política'. Ou seja, o tempo entre a sua publicação e a sua assimilação e operacionalização pelos dirigentes ${ }^{(21)}$.

Porém, a qualidade e a continuidade de uma política pública dependem das pressões e contrapressões feitas pelos diferentes grupos de interesse existentes na sociedade. Entretanto, é necessário que esses atores se apossem do conteúdo de tais políticas para que essas sejam legitimadas.

Em relação ao cenário do local do estudo, a intersetorialidade pode ser incompreendida, ou mesmo deixada em segundo plano, por profissionais de saúde que desconhecem a potencialidade desta ferramenta no enfrentamento do consumo de álcool e outras drogas. Essa apatia pode ser consequência da ausência de estudos que abordem a temática e/ou falta de repasse de experiências bem sucedidas obtidas por estados e municípios, que encorajem a multiplicação em outras localidades. Para o Ministério da Saúde, essa divulgação é necessária ${ }^{(5)}$.

A prevenção voltada para o uso abusivo e/ou dependência de álcool e outras drogas mostrou certa incipiência. Apesar dos gestores vislumbrarem intervenções efetivas, os executores do cuidado carecem de ações voltadas para a redução dos fatores de vulnerabilidade e risco específicos, e fortalecimento dos fatores de proteção. Desta forma, há a necessidade da inserção no ambiente das práticas propostas, para facilitar processos que levem à redução da iniciação no consumo, e das consequências do uso em padrões de maior acometimento global. Para tanto, a lógica da redução de danos deve ser considerada como estratégica ao planejamento de propostas e ações preventivas $^{(5)}$.

No caso específico ao uso das drogas, a finalidade dessas ações é atuar sobre fatores que predispõem o seu uso ou abuso, criando uma mentalidade preventiva ${ }^{(1)}$. Os indivíduos que já apresentam padrão de dependência para uso de drogas não constituem a maior parcela da população de usuários. Desta forma, evidencia-se o importante papel da equipe multiprofissional da ESF enquanto vigilante de problemas associados ao uso de álcool e/ou outras drogas ${ }^{(22)}$.

A promoção da saúde impõe-se pela complexidade dos problemas que caracterizam a realidade sanitária, em que predominam as doenças crônicas não transmissíveis ao lado de doenças sociais contemporâneas, como as violências e as novas endemias. Configura-se também pela potencialidade de estratégias que superem a cultura da medicalização e diminuição de encaminhamentos de pacientes menos graves para a atenção especializada ${ }^{(5)}$.

Os profissionais do CAPS lidam, diariamente, com limitações que impedem que seja realizado um trabalho efetivo junto à comunidade, como a falta de recursos para sair do serviço. Esse fatores contribuem para um modelo de assistência centrado no contexto interno na instituição ${ }^{(23)}$. Entretanto, é fundamental romper barreiras para que as equipes do CAPS e da ESF trabalhem juntas, em sincronia com a atenção psicossocial.

Considerando a existência de espaços com desigualdades de acesso às políticas sociais, os profissionais de saúde devem utilizar estratégias para acolher o indivíduo em uso/abuso de drogas dentro da unidade de saúde. E, promover atendimento individualizado, que respeite a sua autonomia, e sua inserção sócio cultural, de tal modo que consiga estabelecer a vinculação ao serviço e garanta o retorno deste individuo ao serviço quando da necessidade de novo atendimento.

No presente estudo, verificou-se a valorização do envolvimento da família no atendimento ao individuo em uso/abuso de drogas. A configuração de um ambiente favorável à adoção de comportamentos 
prejudiciais à saúde pelos jovens, como o consumo de substâncias psicoativas, é influenciada por uma série de fatores, sendo a família um dos mais importantes ${ }^{(24)}$.

\section{CONSIDERAÇÕES FINAIS}

Concluiu-se que, de forma global, o grau de implantação das diretrizes avaliadas foi insatisfatório, com percentual de concordância de $66,9 \%$ e variação de 65,6\% para a diretriz Prevenção de Agravos e de 68,3\% para a diretriz Promoção e Proteção à Saúde. A avaliação do grau de implantação, segundo os gestores e as categorias profissionais, revelou variações. Para os gestores e os profissionais de saúde do NASF, o grau de implantação foi considerado aceitável - percentual de concordância de $91,5 \%$ e $80,5 \%$, respectivamente. Porém, para os profissionais de saúde das equipes de Saúde da Família, executores diretos do cuidado, o grau de implantação foi insatisfatório, com percentual de concordância de $60,4 \%$.

A existência de relativa rotatividade entre os profissionais, o pouco tempo de atividade em conjunto com a equipe e com a comunidade, e a alta demanda da Unidade podem ter influenciado o conteúdo das respostas. Muitas respostas tinham cunho pessoal, desvinculadas da realidade local, ou eram breves e sucintas, pela sobrecarga de trabalho.

Os resultados apontam a necessidade de estudos ampliados, que possam subsidiar o aperfeiçoamento da atenção à Saúde Mental, e que possam analisar, entre outros aspectos, o papel dos diferentes equipamentos de saúde que compõem a rede de saúde, considerando que a atenção integral é constituída por um conjunto de dispositivos intersetoriais.

\section{REFERÊNCIAS}

1. Büchele F, Coelho EBS, Lindner SR. A promoção da saúde enquanto estratégia de prevenção ao uso das drogas. Ciênc. saúde colet. [Internet] 2009;14(1) [acesso em 22 nov 2012]. Disponível: http://www.scielo.br/scielo. php? pid $=$ S1413-81232009000100033\&script $=$ sci abstract\&tlng=pt

2. Spricigo JS, Carraro TE, Cartana MHF, Reibnitz KS. Atenção ao usuário de drogas - um espaço para o enfermeiro. Texto Contexto-Enferm. [Internet] 2004;13(2) [acesso em 22 nov 2012]. Disponível: http:// redalyc.uaemex.mx/pdf/714/71413215.pdf

3. United Nations Office on Drugs and Crime (UNODC).
Word Drugs Report 2010. [Internet]. [acesso em 25 jun 2010]. Disponível: http://www.unodc.org/documents/ frontpage/UNODC_Annual_Report_2010_LowRes.pdf

4. Galduróz JCF. Epidemiologia do uso de substâncias psicotrópicas no Brasil: dados recentes. In: Prevenção ao uso indevido de drogas: capacitação para conselheiros e lideranças comunitárias. $4^{\mathrm{a}}$ ed. Brasília: Ministério da Justiça. Secretária Nacional de Políticas Sobre Drogas - SENAD; 2011, p. 56-72.

5. Ministério da Saúde (BR). A política do Ministério da Saúde para atenção integral a usuários de álcool e outras drogas. Brasília: CN-DST/AIDS; 2004.

6. Ministério da Saúde (BR). Sistema único de saúde. Conselho nacional de saúde. Comissão Organizadora da III CNSM. Relatório Final da III Conferência Nacional de Saúde Mental. Brasília, 11 a 15 de dezembro de 2001. Brasília: Conselho Nacional de Saúde/Ministério da Saúde; 2002.

7. World health organization (WHO). Ten recommendations for action. Genebra: WHO; 2002.

8. Ministério da Saúde (BR). Secretaria Executiva. Secretaria de Atenção à Saúde. Legislação em Saúde Mental: 1990-2004. Brasília, Ministério da Saúde; 2004.

9. Barros MA, Pillon SC. Programa de Saúde da Família: desafios e potencialidades frente ao uso de drogas. Rev. Eletr. Enf. [Internet] 2006;8(1) [acesso em 13 set 2012]. Disponível: http://www.revistas.ufg.br/index.php/fen/ article/view/932/1129

10. Gonçalves AM. Cuidados diante do abuso e da dependência de drogas: um desafio da prática do programa saúde da família [tese]. Ribeirão Preto (SP): Universidade Estadual de São Paulo; 2002.

11. Souza LGS, Menandro MCS. Atenção primária à saúde: diretrizes, desafios e recomendações. Revisão de bibliografia internacional. Physis [Internet] 2011;21(2) [acesso em 17 set 2012]. Disponível: http://www. scielo.br/scielo.php?script $=$ sci_isoref\&pid $=$ S0103$73312011000200010 \& \operatorname{lng}=$ en\&tlng $=p t$

12. Facchini LA, Piccini RX,Tomasi E, Thumé E, Teixeira VA, Silveira da Silva D, et al. Avaliação da efetividade da Atenção Básica a Saúde em municípios das regiões Sul e Nordeste do Brasil: contribuições metodológicas. Cad. Saúde Pública [Internet] 2008; 24(suppl. 1) [acesso em 3 ago 2012] Disponível: http://www.scielo.br/scielo. php?pid $=$ S0102-311X2008001300020\&script $=$ sci arttext 
13. Silva MOS. Avaliação de políticas e programas sociais: uma reflexão sobre o conteúdo teórico e metodológico da pesquisa avaliativa. In: Silva MOS, organizadora. Pesquisa avaliativa: aspectos teóricos e metodológicos. São Luis, MA:GAEPP (Grupo de Avaliação e Estudo da Pobreza e Políticas Direcionadas à Pobreza), 2008; p.89-178.

14. Hartz ZMA, Silva LMV, organizadoras. Avaliação em saúde: dos modelos conceituais à pratica na análise da Implementação de programas. Salvador: EDUFBA; Rio de Janeiro: Fiocruz, 2005; p. 15-61.

15. Secretaria municipal de Saúde de Maringá. Diagnóstico Social Municipal. [Internet] [acesso em 10 out 2011]. Disponível: http://www.kairos.srv.br/

16. Secretaria de Assistência Social e Cidadania. Diretoria de Programas sobre Drogas. Relatório circunstanciado sobre ações de prevenção e tratamento do uso de drogas na cidade de Maringá, 2010.

17. Cosendey MAE, Hartz ZMA, Bermudez JAZ. Validation of a tool for assessing the quality of pharmaceutical services. Cad. Saúde Pública [Internet] 2003;19(2) [acesso em 4 ago 2012]. Disponível: http://www.scielo.br/scielo.php?pid=S0102311X2003000200006\&script=sci_arttext

18. Oliveira MA, Esher AFSC, Santos EM, Cosendey MAE, Luiza VL, Bermudez JAZ. Avaliação da assistência farmacêutica às pessoas vivendo com HIV/ Aids no município do Rio de Janeiro. Cad. Saúde Pública [Internet] 2002;18(5) [acesso em 4 ago 2012]. Disponível: http://www.scielo.br/scielo.php?pid=S0102311X2002000500036\&script=sci_arttext

19. Tomasi E. Perfil sócio-demográfico e epidemiológico dos trabalhadores da atenção básica à saúde nas regiões Sul e Nordeste do Brasil. Cad. Saúde Pública [Internet] 2008;Suppl 1(24) [acesso em 4 ago 2012]. Disponível: http://www.scielo.br/scielo.php?script=sci_ arttext\&pid=S0102-311X2008001300023\&lng=pt

20. Houaiss, A. Dicionário de língua portuguesa. Rio de Janeiro: Objetiva, 2007.

21. Exworthy M, Blane D, Marmot M. Tackling health inequalities in the United Kingdom: the progress and pitfalls of policy. Health Serv. Res. [Internet] 2003;38(6) [acesso em 12 out 2012]. Disponível: http://www.ncbi. nlm.nih.gov/pmc/articles/PMC1360979/

22. Ministério da Saúde (BR). Secretaria de Atenção à Saúde. Coordenação Geral de Saúde Mental e Coordenação Geral de Atenção Básica. Saúde Mental e Atenção Básica. O vínculo e o diálogo necessários. Brasil: Ministério da Saúde; 2003.

23. Pinho LB, Hernández AMB, Kantorski LP. Serviço substitutivo de saúde mental e inclusão no território: contradições e potencialidades. Cienc Cuid Saude [internet] 2010;9(1) [acesso em 4 set 2012]. Disponível: http://periodicos.uem.br/ojs/index.php/ CiencCuidSaude/article/view/6824/5733

24. Ferreira FGKY, Luz JA, Obrzut Neto L, Santos KA. Uma visão multiprofissional humanizada no tratamento da pessoa com dependência química em enfermaria psiquiátrica de um hospital geral no Paraná. Cogitare enferm. [Internet] 2005;10(2) [acesso em 12 out 2012]. Disponível: http://ojs.c3sl.ufpr.br/ojs2/index.php/ cogitare/article/view/5009/3787

Cogitare Enferm. 2013 Jan/Mar; 18(1):21-8 